\title{
Energy-efficiency Optimization Schemes Based on SWIPT in Distributed Antenna Systems
}

\author{
Weiye $\mathrm{Xu}^{1,3^{*}}$, Junya $\mathrm{Chu}^{2}$, Xiangbin $\mathrm{Yu}^{2}$, and Huiyu Zhou ${ }^{3}$ \\ ${ }^{1}$ School of Information and Communication Engineering, Nanjing Institute of Technology \\ Nanjing, China 211167 \\ [e-mail: xuweiye2014@ hotmail.com] \\ ${ }^{2}$ College of Electronic and Information Engineering, Nanjing University of Aeronautics and Astronautics \\ Nanjing, China 210016 \\ [e-mail: chujynuaa@163.com,yxbxwy@gmail.com] \\ ${ }^{3}$ School of Informatics, University of Leicester \\ Leicester, United Kingdom LE1 7RH \\ [e-mail: hz143@leicester.ac.uk] \\ *Corresponding author :Weiye Xu
}

Received September 15, 2020; revised December 10, 2020; accepted January 18, 2021; published February 28, 2021

\begin{abstract}
In this paper, we intend to study the energy efficiency (EE) optimization for a simultaneous wireless information and power transfer (SWIPT)-based distributed antenna system (DAS). Firstly, a DAS-SWIPT model is formulated, whose goal is to maximize the EE of the system. Next, we propose an optimal resource allocation method by means of the Karush-Kuhn-Tucker condition as well as an ergodic method. Considering the complexity of the ergodic method, a suboptimal scheme with lower complexity is proposed by using an antenna selection scheme. Numerical results illustrate that our suboptimal method is able to achieve satisfactory performance of EE similar to an optimal one while reducing the calculation complexity.
\end{abstract}

Keywords: Distributed Antenna System, Energy Efficiency, Antenna Selection, Simultaneous Wireless Information, Power Transfer, Low Complexity

\footnotetext{
This work is supported in part by Natural Science Research Projects in Jiangsu Higher Education Institutions (18KJB510018), On-Job Training Doctoral Research Fund of Nanjing Institute of Technology (ZKJ201904), and National Natural Science Foundation of China (61571225).
} 


\section{Introduction}

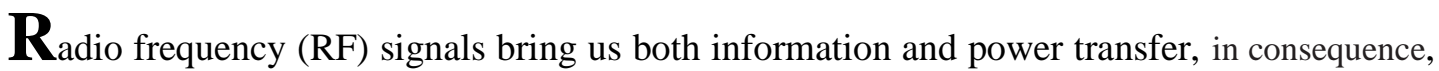
simultaneous wireless information and power transfer (SWIPT) has received more attention.

In [1], the concept of SWIPT was presented at first time, besides, the performance tradeoff between the transferred power and an information transmission rate was studied over flat fading channels [1] and frequency-selective channels [2].

Recently, high data rate demand incurs higher power consumption in the entire network. Therefore, energy efficiency (EE), which is defined as the ratio of spectral efficiency against the total power consumption measured in bit/Hz/Joule, has emerged as an important index of the green wireless communication systems $[3,4]$. In [5] and [6], people have started to investigate the outage probabilities of a joint transmission coordinated multipoint cooperative non-orthogonal multiple access network with SWIPT and a downlink SWIPT-based orthogonal frequency division multiplexing (OFDM) system for multi-user. [5] showed the derivation of the closed-form approximate outage probabilities of users, and [6] optimized the resource allocation (RA) for minimizing the fraction of the outage over all the users. In order to maximize the EE in the coordinated multi-cell multiuser downlink system, the joint base station association and the beamforming scheme are designed based on a successive convex approximation method [7]. In [8], secure transmission of SWIPT Internet of Things (IoT) based multiuser OFDM system with full-duplex IoT devices was examined, and the system can achieve better secrecy performance than the counterpart TDMA system. To maximize the system EE of heterogeneous networks with SWIPT, the authors in [9] looked into the beamforming design and RA, respectively. In [10], the authors presented an energy-efficient optimization scheme for joint power splitting (PS) and beamforming design under the signal-to-interference-plus-noise (SINR) constraints and energy harvesting (EH) constraints of a multiple-input-single-output (MISO) system with SWIPT. However, in the transfer of energy, SWIPT suffers from the fast decay caused by the propagation distances. In other words, the distance will become a challenge during the combination of SWIPT and conventional co-located multiple antenna technique. Interestingly, distributed antenna system (DAS) can avoid the distance limit in SWIPT [11]. This is because, the remote antenna units (RAU) in the cells are distributed more randomly, which reduces the distance between the user and the nearby RAU largely, making SWIPT adaptable. [12] reported a RA scheme to maximize transferred power of DAS-SWIP. [13] introduced a power management strategy for wireless information transfer (WIT) maximization problem under the wireless energy transfer (WET) constraint making use of smart grid. [14] proposes two algorithms for maximizing EE of DAS with SWIPT respectively in single Internet of Things (IoT) device case and multiple IoT devices case. Based on [14], for single IoT device, [15] presented an energy-efficient RA algorithm which has low complexity by means of the Lambert function and the Karush-Kuhn-Tucker (KKT) condition. However, neither of the above two papers considers energy collection in the total energy consumption of the system, nor the noise generated in the information processing, so the system model is not satisfactory.

Based on the issues mentioned above, we will look at the energy-efficient RA of SWIPT-DAS in this paper, and propose energy-efficient RA schemes to achieve system performance improvement. Major contributions of this paper are summarized as following:

1) A new DAS-SWIPT is established, and according to the system model, the achievable rate and system EE are respectively derived for performance optimization. Using these results, the optimization solution can be obtained by the purpose of maximizing EE under the maximum transmit power constraints of each RAU as well as the minimum EH constraint. 
2) Considering the complexity of the optimization problem, we firstly optimize the power allocation (PA) given the PS ratio. Based on the theoretical analysis, a generalized form of the optimal power allocation solution is deduced via the KKT condition. Then, three Corollaries and one Lemma are used to look for the optimal PA coefficients. Finally, the optimal solution of the objective optimization function is sought using the PS ratio, and the corresponding energy-efficient optimal RA algorithm is presented.

3) The RA algorithm needs to compute the PS ratio, and thus it has relatively higher complexity. For this reason, a suboptimal RA algorithm, which has low complexity, is proposed by antenna selection, and corresponding algorithm is also presented. It is shown that the suboptimal algorithm can achieve similar EE performance as that of the optimal algorithm but with lower complexity. Simulation result validates the rational of two schemes proposed in this paper.

The rest of this paper is organized as follows. The system model that targets the problem for EE maximization of downlink SWIPT-based DAS is introduced in Section 2. Section 3 presents an optimal method and a suboptimal method that reduces the calculation complexity to allocate the available power of each RAU and to achieve optimal PS ratio efficiently. Section 4 reports computational simulation results and Section 5 gives the main summary.

Notations: bold lower-case letters are used to represent the column vectors. $W($.$) is the$ Lambert $\mathrm{W}$ function.

\section{System Model}

We introduce a donwlink DAS working single cell as shown in Fig. 1, in which $N$ RAU (containing central base station (BS) ) and single user are included, and a PS is employed by the user. All RAUs and the user hold single antenna. For easy analysis, the $i$-th RAU is written as $\mathrm{RAU}_{i}$. Assuming that all RAUs and the user are fully aware of channel knowdege, and the power allocation of RAU is controlled centrally. This assumption has also been employed in [9-10, 13-17]. To be more realistic, each RAU can operate independently. Namely, all RAUs have their respective transmit power and corresponding maximum transmission power limitations. Assume that RAUs can share the data of user and work with frequency division duplex (FDD) modes [14-16], hence, the signal received at the user terminal can be written as

$$
y=\sum_{i=1}^{N} \sqrt{p_{i}} h_{i} x_{i}+n
$$

where $p_{i}$ is transmit power of $\mathrm{RAU}_{i} . h_{i}$ denotes the fading channel coefficient between $\mathrm{RAU}_{i}$ and the user, modeled as $h_{i}=g_{i} \sqrt{L_{i} S_{i}}$, where $L_{i}=d_{i}^{-\alpha}$ denotes the path loss between $\mathrm{RAU}_{i}$ and the user, $\alpha$ is a path-loss exponent, $d_{i}$ indicates the distance between the user and $\mathrm{RAU}_{i}$. $S_{i}$ denotes the shadow fading with log-normal distribution between $\mathrm{RAU}_{i}$ and the user. $g_{i}$ is a small-scale channel fading coefficinet between user and $\mathrm{RAU}_{i} . x_{i}$ represents the signal transmitted by the $\mathrm{RAU}_{i}$ and it has average power of $E\left[\left|x_{i}\right|^{2}\right]=1$. The noise $n$ is the additive Gaussian one having zero mean and variance of $\sigma^{2}$. 


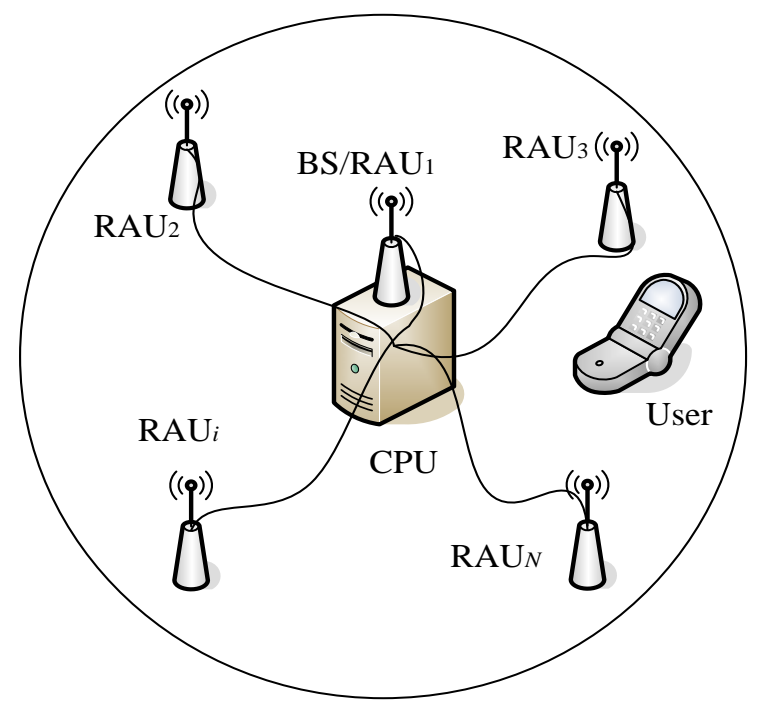

Fig. 1. SWIPT-based disributed antenn system

As illustrated in Fig. 2, at the receiver, the receive signal power segments into two independent parts: a $\rho \in[0,1]$ portion is used for information decoding while the other $(1-\rho)$ portion serves for EH. Thus, the split signal for the WIT of the user can be expressed as

$$
y^{\mathrm{WIT}}=\sqrt{\rho} y+z
$$

where $z$ denotes the Gaussian noise having zero mean and variance $\tau^{2}$ in the course of WIT. Therefore, the split signal for WET is given by

$$
y^{\mathrm{WET}}=\sqrt{1-\rho} y
$$

Accordingly, the power havrvested by the user' EH can be written as

$$
E=\xi(1-\rho)\left[\left(\sum_{i=1}^{N} p_{i} \gamma_{i}\right)+\sigma^{2}\right]
$$

where $\gamma_{i}=L_{i} S_{i}\left|g_{i}\right|^{2}, \xi \in(0,1]$ denotes the conversion efficiencyof the energy. On this basis, the user's achievable rates can be given by

$$
R(\mathbf{p}, \rho)=\log _{2}\left(1+\frac{\rho\left(\sum_{i=1}^{N} p_{i} \gamma_{i}\right)}{\rho \sigma^{2}+\tau^{2}}\right)
$$


where $\mathbf{p}=\left[p_{1}, \ldots, p_{N}\right]^{T}$. With the definiation of EE, the energy efficiency of DAS-SWIPT is

$$
\eta_{E E}(\mathbf{p}, \rho)=\frac{R(\mathbf{p}, \rho)}{T(\mathbf{p}, \rho)}
$$

where $T(\mathbf{p}, \rho)=\sum_{i=1}^{N} p_{i}+p_{c}-E, p_{c}$ represents the circuit power, modeled as [18]

$$
p_{c}=N\left(P_{\mathrm{DAC}}+P_{\mathrm{mix}}+P_{\text {filt }}\right)+2 P_{\mathrm{syn}}+P_{\mathrm{LNA}}+P_{\mathrm{mix}}+P_{\mathrm{IFA}}+P_{\text {filr }}+P_{\mathrm{ADC}}
$$

where $P_{\mathrm{DAC}}, P_{\text {mix }}, P_{\mathrm{LNA}}, P_{\mathrm{IFA}}, P_{\text {filt }}, P_{\text {filr }}, P_{\mathrm{ADC}}$ and $P_{\mathrm{syn}}$ are, respectively, the consumpted powers of digital to analog conversion, mixer, low-noise amplifier, intermediate-frequency amplifier, transmitter-side active filter, receiver-side active filter, and analog to digital conversion as well as frequency synthesizer.

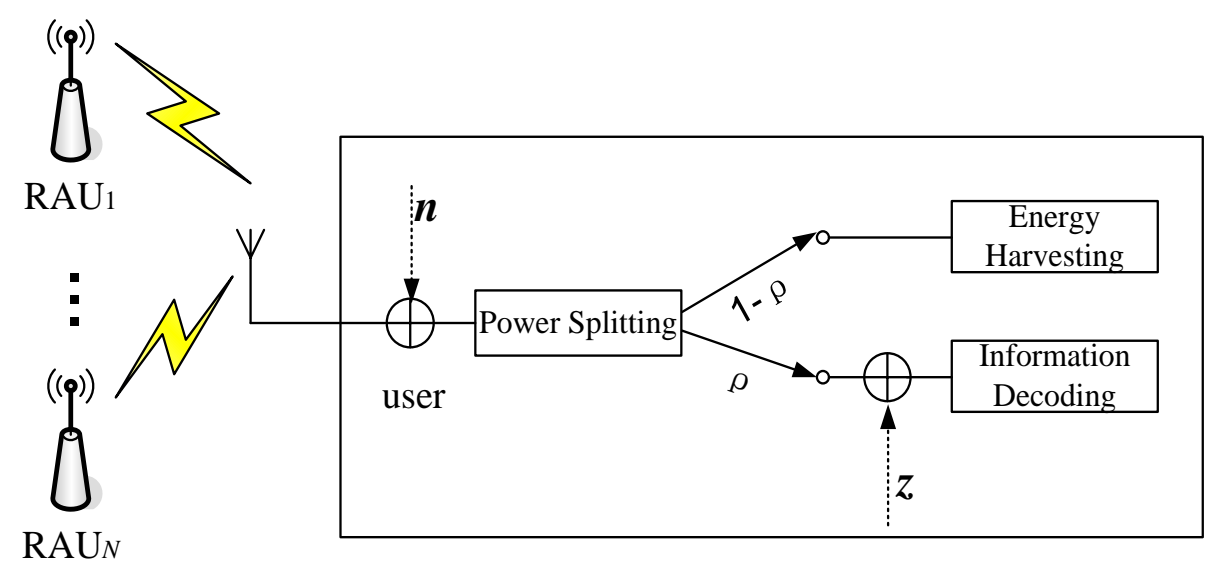

Fig. 2. Diagram of SWIPT [11]

\section{Energy Efficient Resource Allocation}

Here, we will derive an objective function of EE optimization for DAS-SWIPT systems In this section. Then, the general form of the optimal power allocation solution is deduced with the given power splitting ratio by means of the KKT condition. Finally, the optimal solution of the objective optimization function is attained through ergodic the PS ratio, and the corresponding energy efficient optimal resource-allocation scheme and algorithm are presented. Considering the complexity of optimal scheme, a low-complexity suboptimal RA method is developed by using antenna selection technology.

Considering maximum power constraint of each RAU as well as the constraint of minimum energy harvesting, the optimization problem of maximizing EE in (6) can be formulated as 


$$
\begin{gathered}
\max _{\mathbf{p}, \rho} \eta_{E E}(\mathbf{p}, \rho)=\frac{R(\mathbf{p}, \rho)}{T(\mathbf{p}, \rho)}=\frac{\log _{2}\left(1+\frac{\rho\left(\sum_{i=1}^{N} p_{i} \gamma_{i}\right)}{\rho \sigma^{2}+\tau^{2}}\right)}{\sum_{i=1}^{N} p_{i}+p_{c}-E} \\
\text { s.t. } \xi(1-\rho)\left[\left(\sum_{i=1}^{N} p_{i} \gamma_{i}\right)+\sigma^{2}\right] \geq \bar{E}_{0} \\
0 \leq p_{i} \leq P_{\max , i}(i=1, \ldots, N) \\
0 \leq \rho \leq 1
\end{gathered}
$$

where $\bar{E}_{0}$ denotes the minimum energy harvesting, $P_{\max , i}$ is the maximum transmit power of $\mathrm{RAU}_{i}$

\subsection{Optimal RA Scheme}

Firstly, it is observed that (8) can be simplified to a general power allocation problem with fixed $\rho$, expressed as

$$
\begin{gathered}
\max _{\mathbf{p}} \eta_{E E}(\mathbf{p})=\frac{\log _{2}\left(1+\sum_{i=1}^{N} \alpha_{i} p_{i}\right)}{\sum_{i=1}^{N} \beta_{i} p_{i}+\chi} \\
\text { s.t. } \sum_{i=1}^{N} p_{i} \gamma_{i} \geq \Omega \\
0 \leq p_{i} \leq P_{\max , i}(i=1, \ldots, N)
\end{gathered}
$$

where $\alpha_{i}=\rho \gamma_{i} /\left(\rho \sigma^{2}+\tau^{2}\right), \beta_{i}=1-\xi(1-\rho) \gamma_{i}, \chi=p_{c}-\xi(1-\rho) \sigma^{2}$, $\Omega=\bar{E}_{0} /[\xi(1-\rho)]-\sigma^{2}$. For the problem (9), the numerator of the objective function $\eta_{E E}(\mathbf{p})$ is strictly concave and the denominator is linear. Moreover, the constraints are the affine functions on $\left\{p_{i}\right\}$. Hence, (18) becomes a strictly pseudo-convex problem. Thus, (18) will have global optimal solution. Based on this, considering the KKT conditions are sufficient and necessary for the optimality [19,20,13-15], we employ it to address the problem (9).

Without loss of generality, we arrange $\left\{\gamma_{i}, i=1, \ldots, N\right\}$ in descending order as follows

$$
\gamma_{1}>\gamma_{2}>\ldots>\gamma_{N}
$$


The Lagrangian optimization function for (9) is expressed as

$$
L\left(\left\{p_{i}, \mu_{i}, v_{i}, \lambda\right\}\right)=\frac{\log _{2}\left(1+\sum_{i=1}^{N} \alpha_{i} p_{i}\right)}{\sum_{i=1}^{N} \beta_{i} p_{i}+\chi}+\lambda\left(\sum_{i=1}^{N} p_{i} \gamma_{i}-\Omega\right)+\sum_{i=1}^{N} \mu_{i}\left(P_{\mathrm{max}, i}-p_{i}\right)+\sum_{i=1}^{N} v_{i} p_{i}
$$

where $\lambda, \mu_{i}$ and $v_{i}$ are Lagrange multipliers. Utilizing the KKT condition [19], the optimal $\left\{p_{i,}^{*}, \mu_{i}^{*}, v_{i}^{*}, \lambda^{*}\right\}(i=1, \ldots, N)$ must meet the equation below as

$$
\begin{gathered}
\frac{\partial L}{\partial p_{i}}=f_{i}\left(p_{1}^{*}, \ldots, p_{N}^{*}\right)+\lambda^{*} \gamma_{i}+v_{i}^{*}-\mu_{i}^{*}=0 \\
\lambda^{*}\left(\sum_{i=1}^{N} p_{i}^{*} \gamma_{i}-\Omega\right)=0 \\
v_{i}^{*} p_{i}^{*}=0 \\
\mu_{i}^{*}\left(P_{\max , i}-p_{i}^{*}\right)=0 \\
0 \leq p_{i}^{*} \leq P_{\max , i}, \lambda^{*} \geq 0, \mu_{i}^{*} \geq 0, v_{i}^{*} \geq 0
\end{gathered}
$$

where

$$
f_{i}=\frac{\alpha_{i}}{\left(\sum_{j=1}^{N} \beta_{j} p_{j}^{*}+\chi\right)\left(1+\sum_{j=1}^{N} \alpha_{j} p_{j}^{*}\right) \ln 2}-\frac{\beta_{i} \ln \left(1+\sum_{j=1}^{N} \alpha_{j} p_{j}^{*}\right)}{\left(\sum_{j=1}^{N} \beta_{j} p_{j}^{*}+\chi\right)^{2} \ln 2}
$$

Besides, due to the effectiveness of the KKT condition, it has also been employed by some existing references to solve the corresponding EE optimization problem, such as Refs. [13-17, 21, 22], etc.

For convenience, we abandon $p_{i}^{*}$ s from $f_{i}\left(p_{1}^{*}, \ldots, p_{N}^{*}\right)$. Based on (10), $f_{i}+\lambda \gamma_{i}$ is also arranged in a descending order

$$
f_{1}+\lambda \gamma_{1}>f_{2}+\lambda \gamma_{2}>\ldots>f_{N}+\lambda \gamma_{N}
$$

To continue the discussion, several Lemmas are firstly presented below.

Lemma 1: The optimal solution $p_{i}^{*}$ of PA depends on $f_{i}+\lambda \gamma_{i}$ 
Proof: Please see Appendix 1.

$$
\begin{cases}p_{i}^{*}=P_{\max , i} & f_{i}+\lambda^{*} \gamma_{i}>0 \\ 0 \leq p_{i}^{*} \leq P_{\max , i} & f_{i}+\lambda^{*} \gamma_{i}=0 \\ p_{i}^{*}=0 & f_{i}+\lambda^{*} \gamma_{i}<0\end{cases}
$$

According to Lemma 1 and (16), the optimal PA satisfies a general form as follows

$$
\mathbf{P}=\left[P_{\max , 1}, \ldots, P_{\max , N_{0}-1}, p_{N_{0}}^{*}, 0, \ldots, 0\right],
$$

where $p_{N_{0}}^{*} \in\left(0, P_{\max , N_{0}}\right]$.

Considering that the constraint $\sum_{i=1}^{N} p_{i} \gamma_{i} \geq \Omega$, the optimal solution can be divided into $\sum_{i=1}^{N} p_{i} \gamma_{i}=\Omega$ and $\sum_{i=1}^{N} p_{i} \gamma_{i}>\Omega$. When $\sum_{i=1}^{N} p_{i} \gamma_{i}=\Omega$, with (17), then the optimal solution is given as

$$
\begin{aligned}
& N_{0}=\max _{1 \leq j \leq N}\left\{j: \sum_{i=1}^{j-1} \gamma_{i} P_{\max , i}<\Omega\right\} \\
& p_{i}^{*}=P_{\max , i}, \quad 1 \leq i<N_{0} \\
& p_{N_{0}}^{*}=\left(\Omega-\sum_{i=1}^{N_{0}-1} \gamma_{i} P_{\max , i}\right) / \gamma_{N_{0}}
\end{aligned}
$$

When $\sum_{i=1}^{N} p_{i} \gamma_{i}>\Omega$, with (17), (9) is transformed into the following piecewise one

$$
\eta_{E E}\left(k, p_{k}\right)=\left\{\begin{array}{cc}
V_{1}\left(p_{1}\right) & k=1 \\
V_{2}\left(p_{2}\right) & k=2 \\
\ldots & \\
V_{N}\left(p_{N}\right) & k=N
\end{array},\right.
$$

where

$$
V_{k}\left(p_{k}\right)=\frac{\log _{2}\left(1+\sum_{i=1}^{k-1} \alpha_{i} P_{\mathrm{max}, i}+\alpha_{k} p_{k}\right)}{\sum_{i=1}^{k-1} \beta_{i} P_{\mathrm{max}, i}+\beta_{k} p_{k}+\chi}
$$

Due to $V_{k}\left(P_{\max , k}\right)=V_{k+1}(0)$, then (19) is continuous. The derivative of (20) with respect to (w.r.t.) $p_{k}$ gives

$$
V_{k}^{\prime}\left(p_{k}\right)=\frac{\alpha_{k} \Delta-\beta_{k} \Phi \ln \Phi}{\Phi \Delta^{2} \ln 2}=\frac{A_{k}\left(p_{k}\right)}{B_{k}\left(p_{k}\right)},
$$


where $\Phi=\left(1+\sum_{i=1}^{k-1} \alpha_{i} P_{\max , i}+\alpha_{k} p_{k}\right), \Delta=\left(\sum_{i=1}^{k-1} \beta_{i} P_{\max , i}+\beta_{k} p_{k}+\chi\right)$. It is obvious that $B_{k}\left(p_{k}\right)>0$, and the sign of $V_{k}^{\prime}\left(p_{k}\right)$ is consistent with the sign of $A_{k}\left(p_{k}\right)$. Thus, the derivative of numerator $A_{k}\left(p_{k}\right)$ in (21) w.r.t $p_{k}$ gives

$$
A_{k}^{\prime}\left(p_{k}\right)=-\alpha_{k} \beta_{k} \ln \Phi<0
$$

It means that $A_{k}\left(p_{k}\right)$ decreases in each segment, and $V_{k}^{\prime}\left(p_{k}\right)$ is also decreasing in each segment. Besides,

$$
\begin{aligned}
& A_{k}\left(P_{\max , k}\right)=\alpha_{k}\left(\sum_{i=1}^{k} \beta_{i} P_{\max , i}+\chi\right) \\
& -\beta_{k}\left(1+\sum_{i=1}^{k} \alpha_{i} P_{\max , i}\right) \ln \left(1+\sum_{i=1}^{k} \alpha_{i} P_{\max , i}\right) \\
& A_{k+1}(0)=\alpha_{k+1}\left(\sum_{i=1}^{k} \beta_{i} P_{\max , i}+\chi\right) \\
& -\beta_{k+1}\left(1+\sum_{i=1}^{k} \alpha_{i} P_{\max , i}\right) \ln \left(1+\sum_{i=1}^{k} \alpha_{i} P_{\max , i}\right)
\end{aligned}
$$

Because $\alpha_{k}>\alpha_{k+1}$ and $\beta_{k}<\beta_{k+1}$, it holds $A_{k}\left(P_{\max , k}\right)>A_{k+1}(0)$, with $B_{k}\left(P_{\max , k}\right)=B_{k+1}(0)$, we can obtain that $V_{k}^{\prime}\left(P_{\max , k}\right)>V_{k+1}^{\prime}(0)$, namely $V_{k}^{\prime}\left(p_{k}\right)$ is continuously decreasing in throughout the definition domain of piecewise functions. Based on this, Fig. 3 shows two cases of $V_{k}^{\prime}\left(p_{k}\right)$.

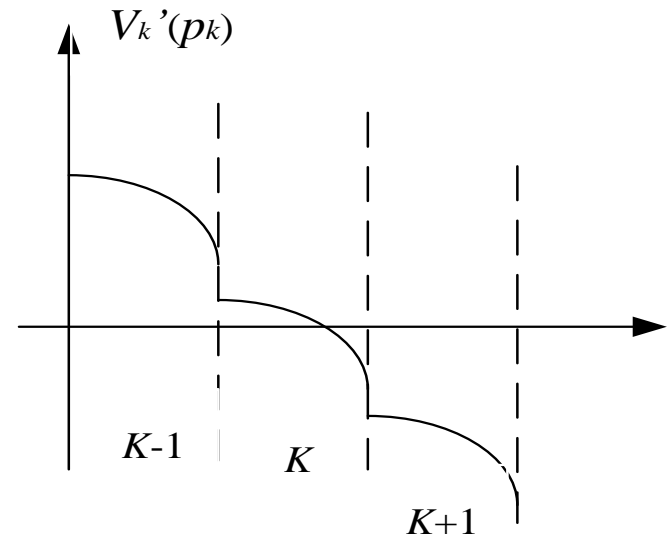

(a)

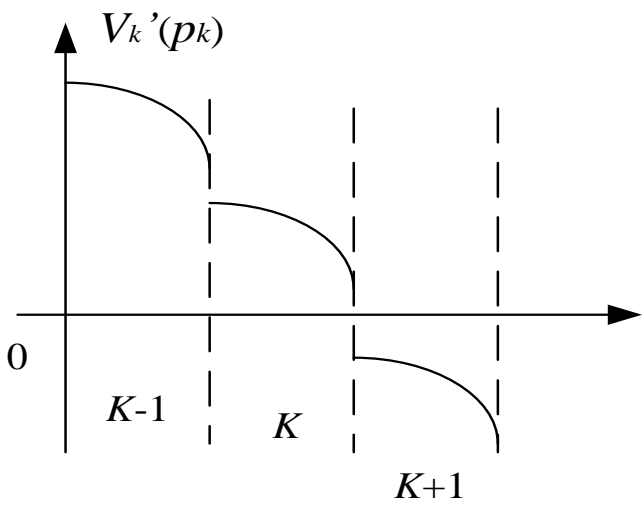

(b)

Fig. 3. Two cases of $V_{k}^{\prime}\left(p_{k}\right)$ 
According to Fig. 3, it can obtain the following Corollaries.

Corollary 1: $N_{0}$ can be given as $N_{0}=\max _{k=1, \ldots, N}\left\{k: V_{k}^{\prime}(0)>0\right\}$.

Proof: Detailed proof please refer to Appendix 2.

Corollary 2: If $A_{N_{0}}\left(P_{\max , N_{0}}\right) \geq 0$, then $p_{N_{0}}^{*}=P_{\max , N_{0}}$.

Proof: Detailed proof please refer to Appendix 3.

Corollary 3: If $A_{N_{0}}\left(P_{\max , N_{0}}\right)<0$, then the optimal $p_{N_{0}}^{*}$ is attained by

$$
p_{N_{0}}^{*}=\frac{1}{\alpha_{N_{0}}}\left[\exp \left\{W\left(\frac{\phi_{1}-\phi_{2}}{e}\right)+1\right\}-\phi_{2}\right]
$$

where $\phi_{1}=\frac{\alpha_{N_{0}}}{\beta_{N_{0}}}\left(\sum_{i=1}^{N_{0}-1} \beta_{i} P_{\max , i}+\chi\right), \phi_{2}=1+\sum_{i=1}^{N_{0}-1} \alpha_{i} P_{\max , i}$.

Proof: Please see Appendix 4 for proof.

According to the above analysis, when the PS ratio $\rho$ is fixed, it is easy to acquire the optimal solution of (9). Thus, the obtained PA is also the implicit function on $\rho$, which will make the optimization problem (8) become much complicated. In other words, it will become very difficult to obtain the optimal $\rho$ directly by optimizing the problem (8). For this reason, we resort to one-dimensional (1D) ergodic method to obtain the optimal $\rho$ considering that it is easy to handle. As a result, the optimal solution of RA in (8) can be attained by the ergodic algorithm and (17) as well as three corollaries. Detailed procedure is summarized in the algorithm 1 below.

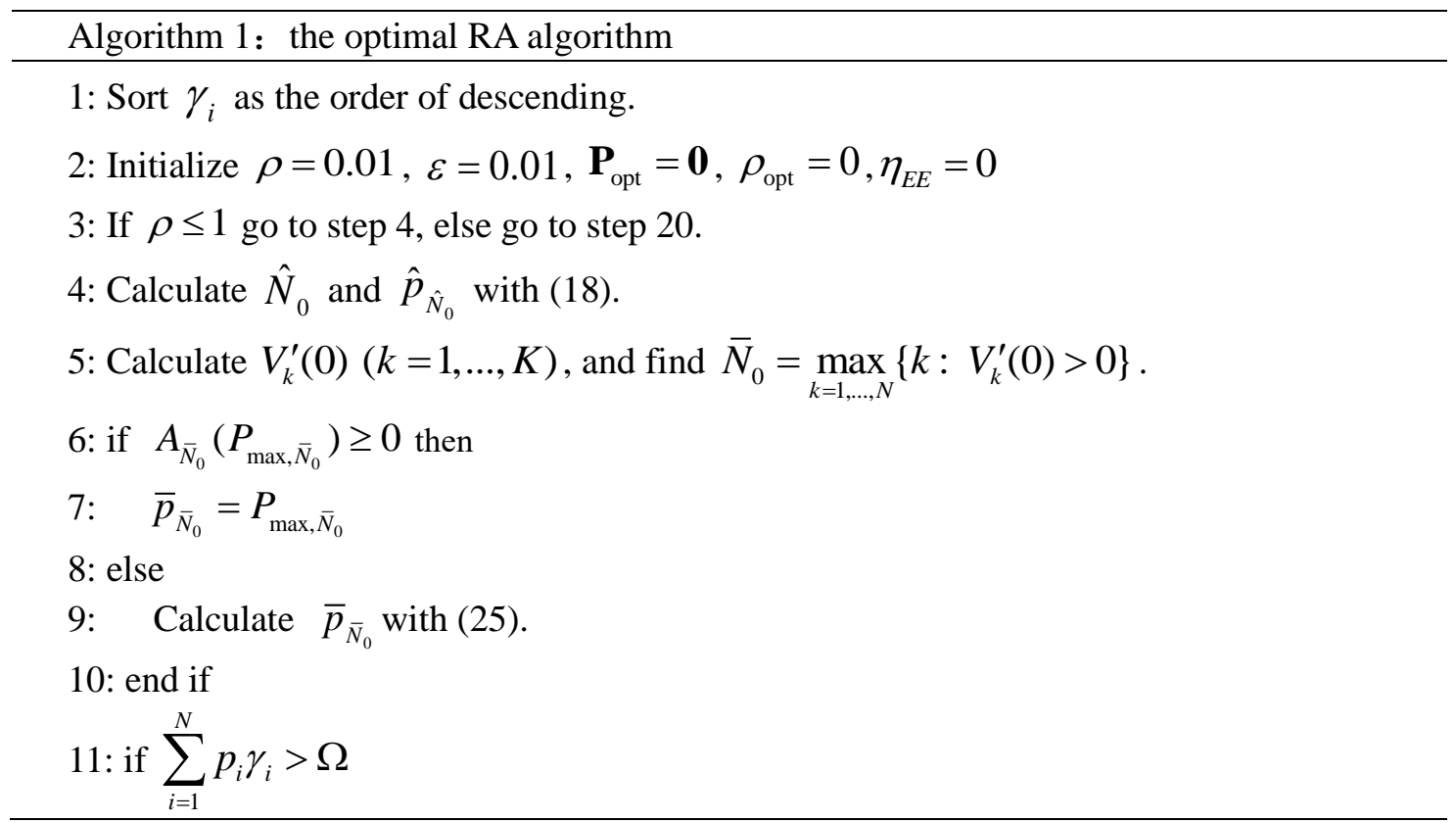


12: $\quad \mathbf{P}=\left[P_{\max , 1}, \ldots, P_{\max , \bar{N}_{0}-1}, \bar{p}_{\bar{N}_{0}}, 0, \ldots, 0\right]$

13: else

14: $\quad \mathbf{P}=\left[P_{\max , 1}, \ldots, P_{\max , \hat{N}_{0}-1}, \hat{p}_{\hat{N}_{0}}, 0, \ldots, 0\right]$

15: end if

16: Calculate $\eta$ with $\rho$ and $\mathbf{P}$.

17: if $\eta>\eta_{E E}$, go to step 18, else go to step 19.

18: $\mathbf{P}_{\mathrm{opt}}=\mathbf{P}, \rho_{\mathrm{opt}}=\rho, \eta_{E E}=\eta$.

19: $q=q+\varepsilon$, go to step 3 .

20: Return $\mathbf{P}_{\mathrm{opt}}$ and $\rho_{\mathrm{opt}}$

\subsection{Suboptimal Resource Allocation Scheme}

Section 3.1 developed the optimal RA solution that needs to ergodic the PS ratio $\rho$, so the complexity is relatively higher. In this subsection, a suboptimal RA solution with low complexity is derived by using antenna selection, and corresponding algorithm 2 is proposed. The suboptimal solution can achieve EE performance similar to that of the optimal solution when the power constraint is applied.

For the DAS, all the data from RAUs to the user terminal will experience the different fading with large scale, such as pass losses and shadow fading since the access distances are not same. Therefore, the RAU nearest to the user terminal will dominate system performance. In terms of the analysis above, utilizing the antenna selection criterion, the RAU with the maximum SNR is selected for transmission. Based on this, a suboptimal RA with single RAU selection is developed. Correspondingly, the received SNR is given by

$$
\gamma_{l}=\max \left\{\gamma_{1}, \ldots, \gamma_{N}\right\}
$$

Then the objective function for maximizing EE can be simplified as

$$
\begin{gathered}
\max _{p_{l}, \rho} \eta_{E E}\left(p_{l}, \rho\right)=\frac{R\left(p_{l}, \rho\right)}{T\left(p_{l}, \rho\right)}=\frac{\log _{2}\left(1+\frac{\rho p_{l} \gamma_{l}}{\rho \sigma^{2}+\tau^{2}}\right)}{p_{l}+p_{c}-\xi(1-\rho)\left(p_{l} \gamma_{l}+\sigma^{2}\right)} \\
\text { s.t. } \xi(1-\rho)\left(p_{l} \gamma_{l}+\sigma^{2}\right) \geq \bar{E}_{0} \\
0 \leq p_{l} \leq P_{\max , l} \\
0 \leq \rho \leq 1
\end{gathered}
$$

According to fractional programming theory, the optimal solution of (27) can be obtained by maximizing the following formula

$$
\max _{p_{l}, \rho} L\left(p_{l}, \rho\right)=R\left(p_{l}, \rho\right)-\lambda T\left(p_{l}, \rho\right)
$$




$$
\begin{gathered}
\text { s.t. } \xi(1-\rho)\left(p_{l} \gamma_{l}+\sigma^{2}\right) \geq \bar{E}_{0} \\
0 \leq p_{l} \leq P_{\max , l} \\
0 \leq \rho \leq 1
\end{gathered}
$$

Obviously, when $\rho$ is fixed, the objective function in (28) becomes concave with linear constraints, written as $P_{l}(\rho) \triangleq\left\{p_{l} \mid l(\rho) \leq p_{l} \leq P_{\max , l}\right\} \quad$, where $l(\rho)=\bar{E}_{0} / \xi(1-\rho) \gamma_{l}-\sigma^{2} / \gamma_{l}$. Thus (28) is equivalent to the following double maximization problem

$$
\max _{0 \leq \rho \leq 1} \max _{p_{l} \in P_{l}(\rho)} L\left(p_{l}, \rho\right)=R\left(p_{l}, \rho\right)-\lambda T\left(p_{l}, \rho\right) .
$$

We can see that the inner maximization problem (IMP) in (29) is convex with fixed $\rho$. Moreover, since $L\left(p_{l}, \rho\right)$ is a concave function on $p_{l}$, there will be a sole solution for the IMP. Let $p_{l}^{*}(\rho)$ stand for the sole optimal solution for IMP in (29), then the optimal PS ratio $\rho$ in (28) is attained by tackling the problem below

$$
\max _{0 \leq \rho \leq 1} \psi(\rho)
$$

where $\psi(\rho) \triangleq R\left(p_{l}^{*}(\rho), \rho\right)-\lambda T\left(p_{l}^{*}(\rho), \rho\right)$.

Next, we solve (30) by analyzing all cases of $p_{l}(\rho)$. When $\rho$ is fixed, the inner maximization problem of (29) can be simplified as

$$
\begin{aligned}
& \max _{p_{l}} L\left(p_{l}, \rho\right)=R\left(p_{l}, \rho\right)-\lambda T\left(p_{l}, \rho\right) \\
& \text { s.t. } l(\rho) \leq p_{l} \leq P_{\text {max }, l}
\end{aligned}
$$

The derivative of (31) w.r.t. $p_{l}$ is

$$
\frac{\partial L\left(p_{l}, \rho\right)}{\partial p_{l}}=\frac{\rho \gamma_{l}}{\left(\rho \sigma^{2}+\tau^{2}+\rho p_{l} \gamma_{l}\right) \ln 2}-\lambda\left[1-\xi(1-\rho) \gamma_{l}\right]
$$

Let $\frac{\partial L\left(p_{l}, \rho\right)}{\partial p_{l}}=0$, and the stationary point of (32) can be obtained as

$$
p_{l}^{s}(\rho)=\frac{1}{\lambda\left[1-\xi(1-\rho) \gamma_{l}\right] \ln 2}-\frac{\rho \sigma^{2}+\tau^{2}}{\rho \gamma_{l}}
$$


The problem (31) is convex under bounded constraints, so its optimal value must be either the endpoint of intervals (i.e., $l(\rho)$ or $\left.P_{\max , l}\right)$ or the stationary point (33). Then, the optimal PA of $(31)$ is

$$
p_{l}^{*}(\rho)= \begin{cases}l(\rho), & \text { if } p_{l}^{s}(\rho) \leq l(\rho) \leq P_{\max , l} \\ p_{l}^{s}(\rho), & \text { if } l(\rho) \leq p_{l}^{s}(\rho) \leq P_{\max , l} \\ P_{\text {max }, l}, & \text { if } l(\rho) \leq P_{\max , l} \leq p_{l}^{s}(\rho)\end{cases}
$$

Next, the above three cases in (34) are considered, and the best $\rho$ is chosen as the optimal solution of (30). Thus, (30) can be expressed as

$$
\max _{\rho \in \Theta} \psi(\rho)
$$

where $\Theta$ stands for the union of the feasible region intervals of $\rho$. For a problem under the constraint of the intervals, the optimal value must be either the endpoint of interval or the stationary point from the optimized function. Based on this, the optimal value of (35) is the point satisfying $d \psi(\rho) / d \rho=0(\rho \in \Theta)$ or the end point of $\Theta$. Then we will analyze the three cases of (34).

1) $p_{l}(\rho)=l(\rho)$, in this case, we can obtain

$$
\psi(\rho)=\log _{2}\left(1+\frac{\rho\left(\frac{\bar{E}_{0}}{\xi(1-\rho)}-\sigma^{2}\right)}{\rho \sigma^{2}+\tau^{2}}\right)-\lambda\left(\frac{\bar{E}_{0}}{\xi(1-\rho) \gamma_{l}}-\frac{\sigma^{2}}{\gamma_{l}}+p_{c}-\bar{E}_{0}\right)
$$

With (36), the derivative of $\psi(\rho)$ w.r.t. $\rho$ can be given by

$$
\frac{d \psi(\rho)}{d \rho}=\frac{\bar{E}_{0} \tau^{2}-\xi \sigma^{2} \tau^{2}(1-\rho)^{2}+\bar{E}_{0} \rho^{2} \sigma^{2}}{(1-\rho)\left(\rho \sigma^{2}+\tau^{2}\right)\left[\bar{E}_{0} \rho+\xi \tau^{2}(1-\rho)\right] \ln 2}-\frac{\lambda \bar{E}_{0}}{\xi \gamma_{l}(1-\rho)^{2}}
$$

Thus, let $\frac{d \psi(\rho)}{d \rho}=0, \rho$ satisfies the following cubic equation

$$
a_{1} \rho^{3}+b_{1} \rho^{2}+c_{1} \rho+d_{1}=0
$$


where

$$
\begin{aligned}
& a_{1}=\xi \gamma_{l} \sigma^{2}\left(\xi \tau^{2}-\bar{E}_{0}\right) \quad, \quad b_{1}=\left(\bar{E}_{0} \xi \gamma_{l}-3 \xi^{2} \gamma_{l} \tau^{2}\right) \sigma^{2}+\lambda \bar{E}_{0} \sigma^{2}\left(\xi \tau^{2}-\bar{E}_{0}\right) \ln 2 \\
& c_{1}=\left(3 \xi^{2} \gamma_{l} \sigma^{2}-\bar{E}_{0} \xi \gamma_{l}\right) \tau^{2}-\lambda \bar{E}_{0} \tau^{2}\left(\xi \sigma^{2}+\bar{E}_{0}+\xi \tau^{2}\right) \ln 2 \\
& d_{1}=\left(\bar{E}_{0} \gamma_{l}-\xi \gamma_{l} \sigma^{2}-\bar{E}_{0} \lambda \tau^{2} \ln 2\right) \xi \tau^{2} \\
& \quad 2) \quad p_{l}(\rho)=p_{l}^{s}(\rho), \text { in this case, we obtain } \\
& \psi(\rho)=\log _{2}\left(\frac{\rho \gamma_{l}}{\lambda\left[1-\xi(1-\rho) \gamma_{l}\right] \ln 2}\right)-\lambda\left\{\frac{1}{\lambda \ln 2}-\frac{\left(\rho \sigma^{2}+\tau^{2}\right)}{\rho \gamma_{l}}+\frac{\xi(1-\rho) \gamma_{l} \tau^{2}}{\rho \gamma_{l}}+p_{c}\right\}
\end{aligned}
$$

By setting the first-order derivative of $\psi(\rho)$ in (39) with respect to $\rho$ as zero, we have the following cubic equation:

$$
a_{2} \rho^{3}+b_{2} \rho^{2}+c_{2} \rho+d_{2}=0
$$

where

$$
a_{2}=\xi \gamma_{l}^{2} \sigma^{2} \quad, \quad b_{2}=\lambda \xi \gamma_{l} \sigma^{2} \tau^{2}\left(1-\xi \gamma_{l}\right) \ln 2
$$

$c_{2}=\tau^{2}\left(1-\xi \gamma_{l}\right)\left[\lambda \sigma^{2}\left(1-\xi \gamma_{l}\right) \ln 2+\lambda \xi \gamma_{l} \tau^{2} \ln 2-\gamma_{l}\right], d_{2}=\lambda \tau^{4}\left(1-\xi \gamma_{l}\right)^{2} \ln 2$.

3) $p_{l}(\rho)=P_{\max , l}$, in this case, we obtain

$$
\psi(\rho)=\log _{2}\left(1+\frac{\rho P_{\text {max }, l} \gamma_{l}}{\rho \sigma^{2}+\tau^{2}}\right)-\lambda\left[P_{\max , l}+p_{c}-\xi(1-\rho)\left(P_{\max , l} \gamma_{l}+\sigma^{2}\right)\right]
$$

By setting the first-order derivative of $\psi(\rho)$ in (41) w.r.t. $\rho$ as zero, we have the following qadratic quation:

$$
a_{3} \rho^{2}+b_{3} \rho+c_{3}=0
$$

where $a_{3}=\lambda \xi \sigma^{2}\left(\sigma^{2}+P_{\text {max }, l} \gamma_{l}\right)^{2} \ln 2, b_{3}=\lambda \xi\left(P_{\text {max }, l} \gamma_{l}+\sigma^{2}\right)\left(2 \sigma^{2} \tau^{2}+P_{\text {max }, l} \gamma_{l} \tau^{2}\right) \ln 2$, $c_{3}=\lambda \xi \tau^{4}\left(P_{\max , l} \gamma_{l}+\sigma^{2}\right) \ln 2-P_{\max , l} \gamma_{l} \tau^{2}$.

To sum up, we can obtain the possible value of $\rho^{*}$ by solving (38), (41) and (44), and then check all the boundary values. Finally, we select one $\rho$ which can maximize $\psi(\rho)$ as the optimal solution $\rho^{*}$ of (28), and the corresponding PA $p_{l}^{*}$ could be acquired by (34). The Dinkelbach method is used to update $\lambda$, and the specific steps are shown in Algorithm 2. 


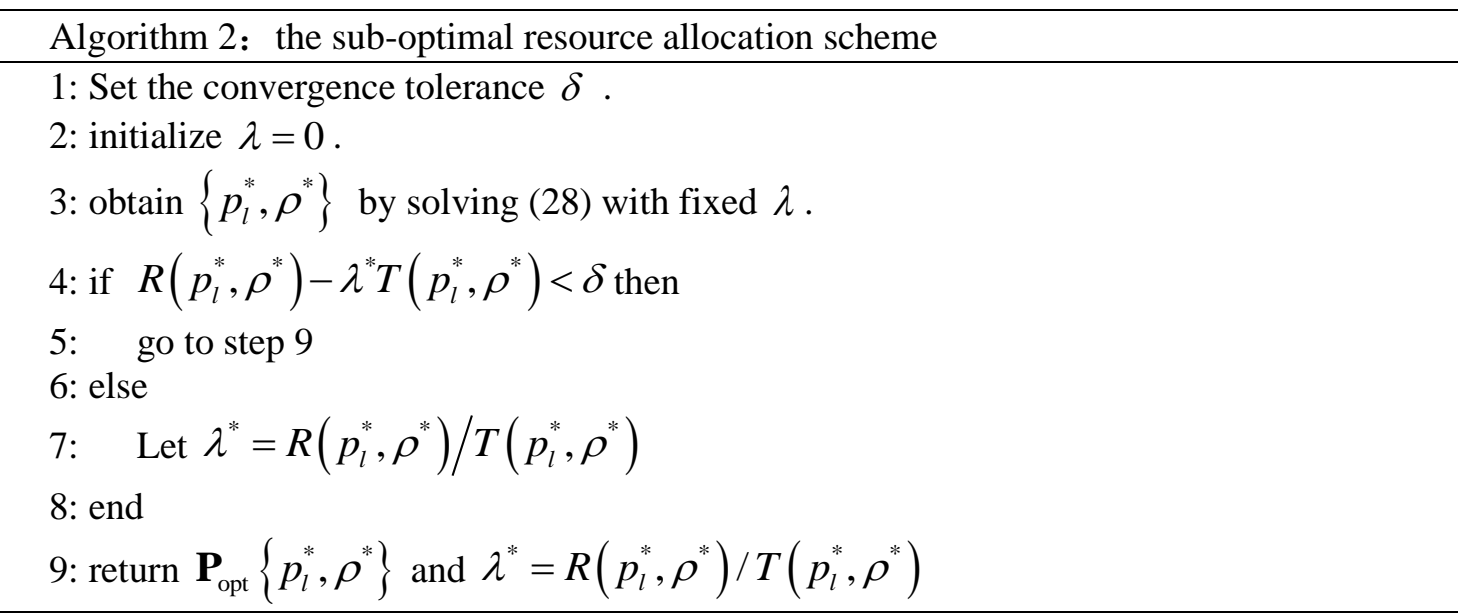

\subsection{Complexity Analysis}

The complexity analysis of two algorithms is given in this subsection. Regarding the Algorithm 1, it mainly involves the 1D search of PS ratio and the calculation of $N$ power allocation coefficients. Hence, the complexity is approximated as $O\left(N I_{1}\right)$, where $I_{1}$ depends on the step size of the $1 \mathrm{D}$ ergodic method, and $O(\cdot)$ denotes the big- $O$ notation. Algorithm 2 mainly involves single-loop iteration of the Dinkelbach method, and corresponding complexity is approximated as $O\left(N I_{2}\right)$, and $I_{2}$ is the iterative number of the Dinkelbach method. According to the analysis above, the Algorithm 1 will have higher complexity than the Algorithm 2 because the former needs to set very small step to search the optimal PS ratio for ensuring the accuracy.

\section{Simulation Result}

The EE performance of the proposed optimal and suboptimal methods in DAS-SWIPT system is hereby evaluated in this section. Unless specified otherwise, in simulation, the main system parameters are illustrated in Table 1. For convenience, we assume that $P_{\max , i}=P_{\max }$. The parameters used in $p_{c}$ are listed in Table 2 [23], and $\tau^{2}=-50 \mathrm{dBm}$.

Table 1. Main parameters

\begin{tabular}{|l|l|}
\hline Cell radius $R$ & $1000 \mathrm{~m}$ \\
\hline Polar coordinate of the BS/RAU & \\
\hline Polar coordinates of other RAUs & $(0,0)$ \\
\hline Standard deviation of the shadow fading & $(2 R / 3,2 \pi i /(N-1)), i=1, \ldots, N-1$ \\
\hline Noise power & $8 \mathrm{~dB}$ \\
\hline Energy conversion efficiency & $\sigma^{2}=-100 \mathrm{dBm}$ \\
\hline Number of RAU & $\xi=0.6$ \\
\hline Minimum energy harvesting & $N=7$ \\
\hline
\end{tabular}


Table 2. Parameters of $p_{c}$

\begin{tabular}{|c|c|}
\hline$P_{\mathrm{DAC}}=15.35 \mathrm{~mW}$ & $P_{\text {filr }}=2.5 \mathrm{~mW}$ \\
\hline$P_{\text {mix }}=30.3 \mathrm{~mW}$ & $P_{\text {filt }}=2.5 \mathrm{~mW}$ \\
\hline$P_{\mathrm{LNA}}=20 \mathrm{~mW}$ & $P_{\mathrm{ADC}}=10 \mathrm{~mW}$ \\
\hline$P_{\mathrm{IFA}}=3 \mathrm{~mW}$ & $P_{\text {syn }}=50 \mathrm{~mW}$ \\
\hline
\end{tabular}

In Fig. 4, we show the EE performance of this system under different energy harvesting constraints, where $\alpha=3, \bar{E}_{0}=-65 \mathrm{dBm},-60 \mathrm{dBm},-55 \mathrm{dBm}$. As is shown in Fig. 4, the EE decreases dramatically when the energy harvesting constraint $\bar{E}_{0}$ increases. The reason is when the EH constraint is relatively high, it is not very likely for the system to obtain the Quality of Service (QoS) levels although all the RAUs have maximum transmit power. When the power constraint $P_{\text {max }, i}$ is small, the system EE is smaller in the suboptimal scheme than in the optimal scheme. This is because the power allocation coefficient in suboptimal scheme become small due to the EH constraints. The suboptimal scheme only selects one RAU, so that it cannot meet the EH constraint. Therefore, the information transmission rate is sacrificed if we increase the energy harvesting, which makes the rate much smaller, and the system EE follows. Moreover, the EE performance gap between the suboptimal and optimal schemes increases with the decrease of the EH constraint. Most of $N_{0}$ are equal to 1 when $P_{\max , i}$ is large, that is to say, only one RAU is needed to satisfy the EH constraint and achieve the best EE performance. At this time, the corresponding RAU can be selected by antenna selection to achieve the same EE as the optimal one. Therefore, the EE performance in suboptimal scheme is consistent in optimal scheme in the case of large $P_{\max , i}$, but the computational complexity in suboptimal scheme can be greatly lowed.

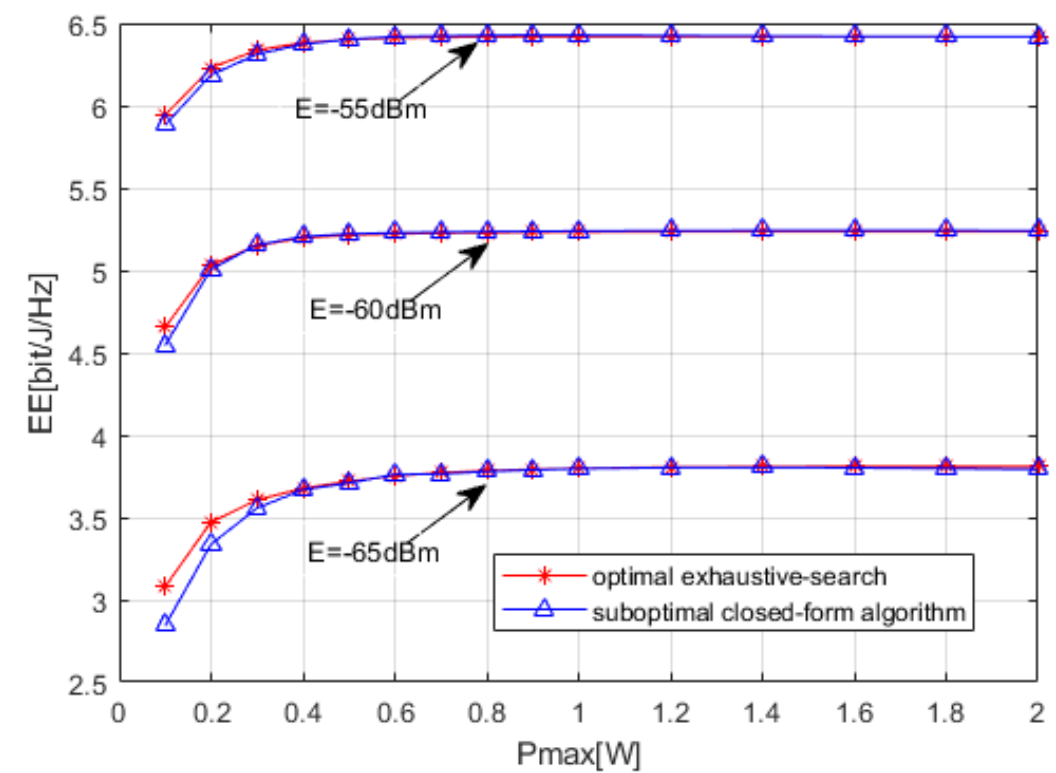

Fig. 4. EE versus $P_{\max }$ of DAS-SWIPT under different energy harvesting constraints 
In Fig. 5, we depict the system EE of the DAS-SWIPT under different numbers of RAU with $\alpha=3$ and $N=5,7$. In this figure, the EE performance in suboptimal RA scheme is similar to that in optimal one, especially when $P_{\max , i}$ is large, the EE performance of the two schemes tends to be the same, which shows that the suboptimal scheme can maintain stability performance with different parameters and has high feasibility. In addition, the system EE increases when the number of RAU increases. On one hand, as $N$ increases, RAU is more densely distributed in the cell, then the wireless access distance between users and RAU will be decreased and the impact of pass loss is reduced, which improves the corresponding system EE. On the other, the increase of the number of RAU can also bring spatial diversity gain. Based on the above two reasons, it can be concluded that the EE performance of this system could be effectively enhanced with the increasing of the number of RAU.

By using antenna selection, the suboptimal RA algorithm transforms the original objective function from a multi-dimensional optimization problem into a two-dimensional optimization problem. So we derived the closed-form solution, which greatly reduces the computational complexity. This can also be seen from the table of running time with two proposed schemes. As shown in Table 3, the time required for the suboptimal scheme is about only of $1 / 25$ the optimal one. Moreover, the running time increases with the increase of $N$ for two schemes. The results above also accord with the complexity analysis in section 3.3. Besides, when $P_{\max , i}$ is large, the system EE obtained by the suboptimal scheme is consistent with the optimal one. Hence, the suboptimal scheme provides a choice for engineering implementation.

Table 3. Comparison of the running time

\begin{tabular}{|c|c|c|}
\hline Number of RAU & Optimal scheme & Suboptimal scheme \\
\hline$N_{t}=7$ & $799.54 \mathrm{~s}$ & $30.1 \mathrm{~s}$ \\
\hline$N_{t}=5$ & $764.26 \mathrm{~s}$ & $27.73 \mathrm{~s}$ \\
\hline
\end{tabular}

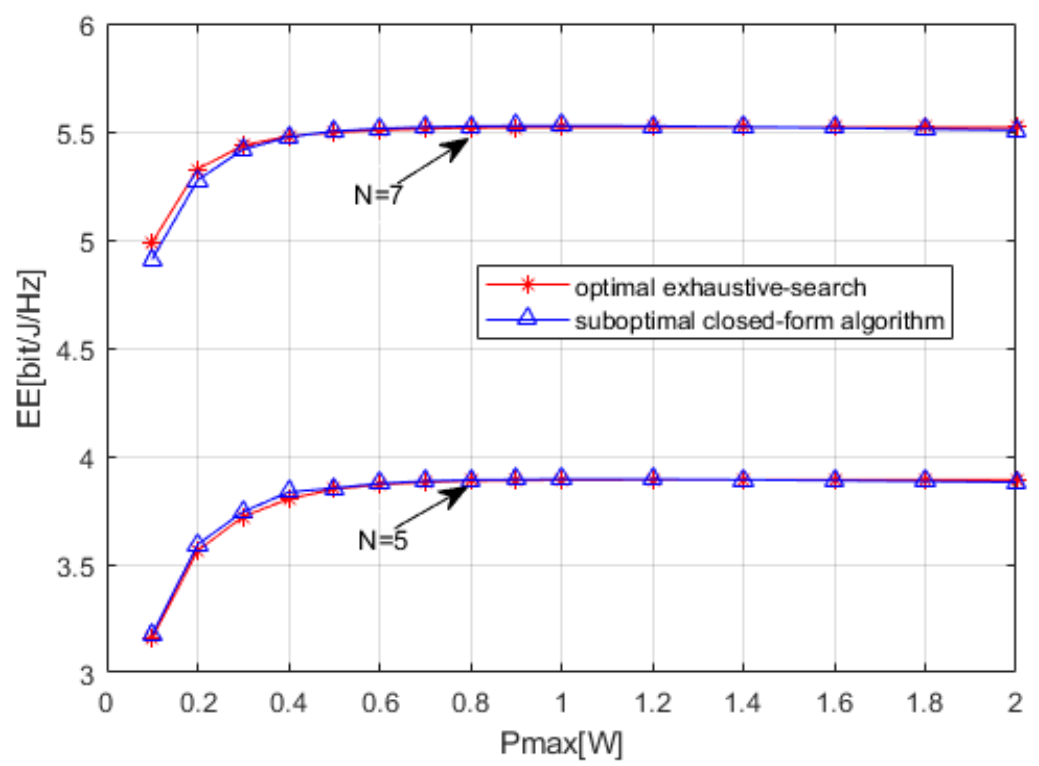

Fig. 5. EE versus $P_{\max }$ of DAS-SWIPT under different $N$ 
Fig. 6 plots the systerm EE performance under different path loss exponents with $\alpha=3,3.2$, $\bar{E}_{0}=-60 \mathrm{dBm}$. From this figure, it can be seen that the EE preformance decreases with the increase of the path loss exponent. This is because the increase of $\alpha$ leads to the increase of path loss, therefore, the EE performance is remarkebally impacted. This is also consistent with the actual communication situation, and further illustrates the rationality of the proposed schemes.

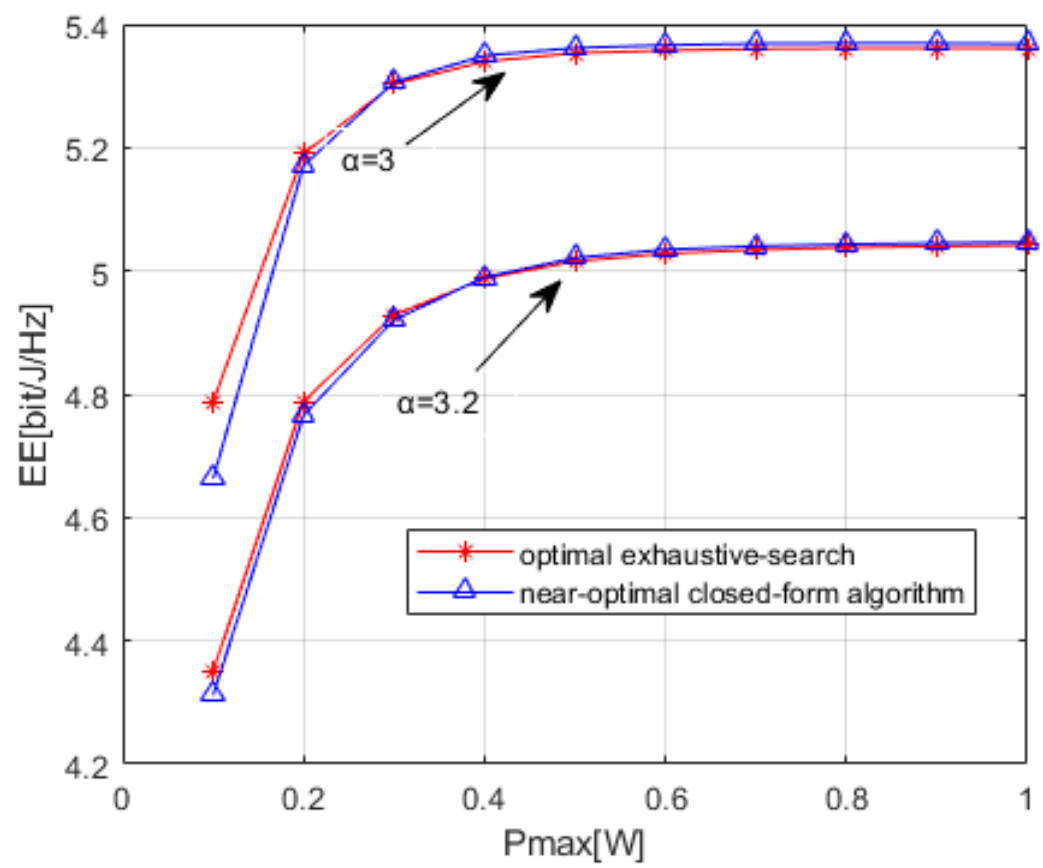

Fig. 6. EE versus $P_{\max }$ for DAS-SWIPT under different $\alpha$

Fig. 7 illustrates the system EE versus $P_{\max }$ with different RA schemes, where the proposed scheme, existing scheme in [14] and the RA scheme under fixed PS ratio $(\rho=0.5)$ are compared, $\alpha=3, \bar{E}_{0}=-50 \mathrm{dBm}, \tau^{2}=0$. For fair comparison with [14], the energy harvesting in the total energy consumption and the noise generated in the information processing are no longer considered. Correspondingly, the optimization objective function is reduced to the EE in [(2), 14]. In Fig. 7, the EE performance of proposed RA scheme is consistent with that of the existing RA scheme since they are both optimal. Moreover, they perform better than the RA scheme with fixed $\rho$, that is, the former two have higher EE than the latter. This is because the latter is based on the fixed PS ratio and does not optimize $\rho$. The results above also provide evidence of the validity of the proposed scheme.

\section{Conclusions}

This paper combines SWIPT with DAS to study the energy efficient RA design in DAS-SWIPT system. Firstly, according to KKT condition and ergodic algorithm, we develop an energy-efficient optimal RA scheme. Then, a sub-optimal RA scheme, which has low complexity, is presented utilizing the antenna selection method. Numerical results show that suboptimal scheme can achieve EE performance similar to that of the optimal scheme, 
especially when the power constraints are large. Moreover, the computational complexity is greatly reduced. Since the perfect channel information is not easy to attain in practice and the system will operate in large-scale antenna environment, in the future work, we will work on the RA scheme design under imperfect channel information and large number of RAU, and it is expected that some valued results can be obtained.

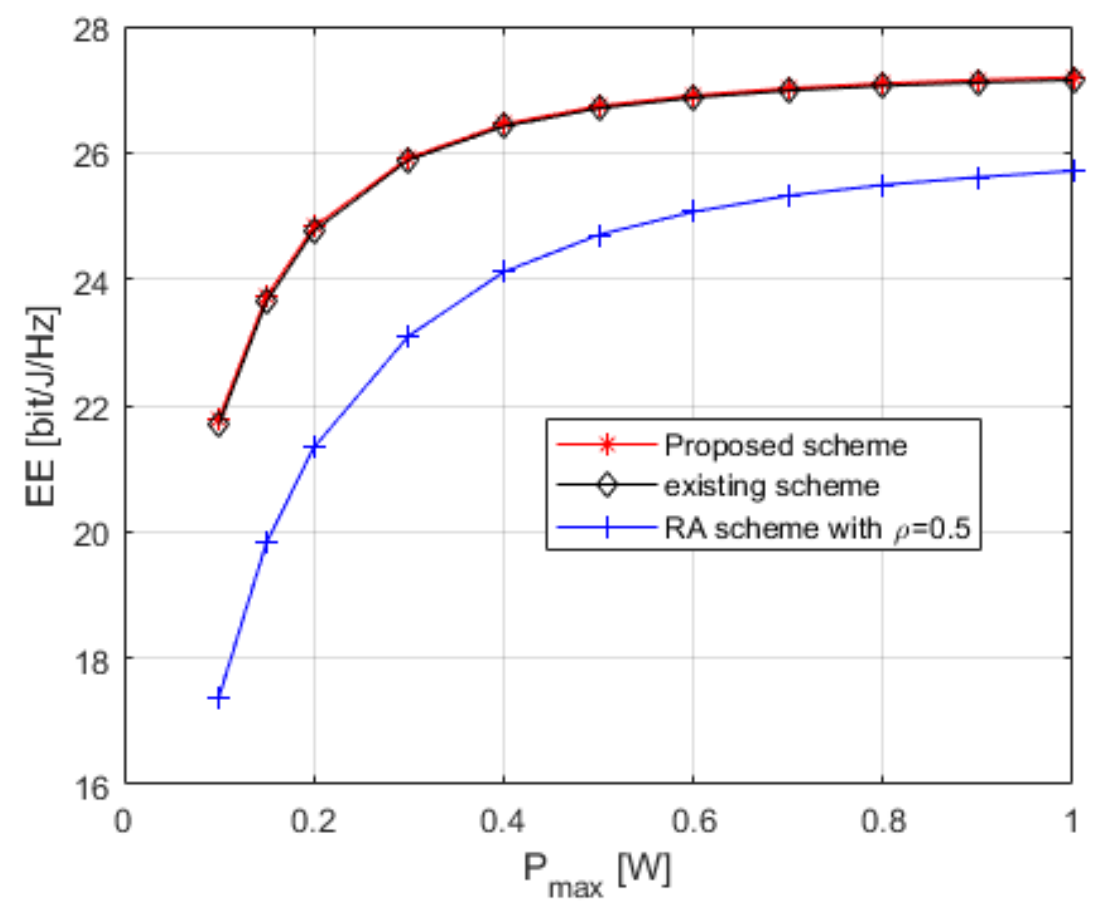

Fig. 7. EE versus $P_{\max }$ for DAS-SWIPT with different schemes

\section{Appendix 1}

In the appendix 1, we give the proof of Lemma 1.

If $f_{i}+\lambda^{*} \gamma_{i}>0$, it holds $f_{i}+\lambda^{*} \gamma_{i}=-v_{i}^{*}+\mu_{i}^{*}>0$ with (12), then $\mu_{i}^{*}>v_{i}^{*} \geq 0$. We can obtain $p_{i}^{*}=P_{\max , i}$ with (13). Similarly, if $f_{i}+\lambda^{*} \gamma_{i}<0$, then $p_{i}^{*}=0$. When $f_{i}+\lambda^{*} \gamma_{i}=0$, then $v_{i}^{*}=\mu_{i}^{*}$, and it is impossible that $p_{i}^{*}$ and $\left(P_{\max , i}-p_{i}^{*}\right)$ equals to zero at the same time. $v_{i}^{*}=\mu_{i}^{*}=0$ and $0 \leq p_{i}^{*} \leq P_{\max , i}$ according to (13).

\section{Appendix 2}

In the appendix 2, the proof on Corollary 1 is provided.

Let $K=\max _{k=1, \ldots, N}\left\{k: V_{k}^{\prime}(0)>0\right\}$. With Fig. 3, it is obvious that $V_{k}^{\prime}\left(p_{k}\right)>0$ when $k<K$, and $V_{k}^{\prime}\left(p_{k}\right)<0$ when $k>K$. Namely, $V_{k}\left(p_{k}\right)$ is increasing when $k<K$ and decreasing when $k>K$. Thus, $N_{0}=K$. 


\section{Appendix 3}

In the appendix 3, the proof on Corollary 2 is provided.

With Corollary 1 , it obtains $N_{0}=\max _{k=1, \ldots, N}\left\{k: V_{k}^{\prime}(0)>0\right\}$, then $A_{N_{0}+1}(0)<0$. If $A_{N_{0}}\left(P_{\max , N_{0}}\right) \geq 0$, shown as (a) in Fig. 3, it holds $V_{k}^{\prime}\left(p_{k}\right)>0$ when $k<N_{0}$, and $V_{k}^{\prime}\left(p_{k}\right)<0$ when $k>N_{0}$. In other words, $V_{k}\left(p_{k}\right)$ is increasing when $k<N_{0}$, and decreasing when $k>N_{0}$, as well as the objective function in (19) is continuous, thus $p_{N_{0}}^{*}=P_{\max , N_{0}}$.

\section{Appendix 4}

In the appendix 4 , the proof on Corollary 3 is provided.

If $A_{N_{0}}\left(P_{\max , N_{0}}\right)<0$, corresponding to (b) in Fig. 3, it follows that $A_{N_{0}}(0)>0$ and $A_{N_{0}}\left(P_{\max , N_{0}}\right)<0$. Hence, $p_{N_{0}}^{*}$ can be solely obtained when $A_{N_{0}}\left(p_{N_{0}}\right)=0$. With the aid of Lambert function, the optimal value of $p_{N o}, p_{N_{0}}^{*}$ is attained as (25) and it is a sole solution.

\section{Acknowledgment}

The authors would like to thank the anonymous reviewers for their valuable comments which improve the quality of this paper greatly.

\section{References}

[1] L. R. Varshney, "Transporting information and energy simultaneously," in Proc. of IEEE International Symposium on Information Theory (ISIT’2008), pp. 1612-1616, 2008. Article (CrossRef Link)

[2] P. Grover and A. Sahai, "Shannon meets Tesla: Wireless information and power transfer," in Proc. of IEEE International Symposium on Information Theory (ISIT'2010), pp. 2363-2367, June 2010. Article (CrossRef Link)

[3] G. Y. Li, Z. Xu, C. Xiong, C. Yang, S. Zhang, Y. Chen, and S. Xu, "Energy-efficient wireless communications: Tutorial, Survey, and Open issues," IEEE Wireless Communications Magazine, vol. 18, no. 6, pp. 28-35, 2011. Article (CrossRef Link)

[4] X. Gao, D. Niyato, P. Wang, K. Yang, and J. An, "Contract design for time resource assignment and pricing in backscatter-assisted RF-powered networks," IEEE Wireless Communication Letters, vol. 9, no. 1, pp. 42-46, 2020. Article (CrossRef Link)

[5] R. Lei and D. Xu, "On the outage performance of JT-CoMP-CNOMA networks with SWIPT," IEEE Communications Letters, 2020. Article (CrossRef Link)

[6] D. Xu and H. Zhu, "Outage minimized resource allocation for multiuser OFDM systems with SWIPT," IEEE Access, vol. 7, pp. 79714-79725, 2019. Article (CrossRef Link)

[7] J. An, Y. Zhang, X. Gao, and K. Yang, "Energy-efficient base station association and beamforming for multi-cell multiuser systems," IEEE Transactions on Wireless Communications, vol. 19, no. 4, pp. 2841-2854, 2020. Article (CrossRef Link)

[8] D. Xu and H. Zhu, "Secure transmission for SWIPT IoT systems with full-duplex IoT devices," IEEE Internet of Things Journal, vol. 6, no. 6, pp. 10915-10933, 2019. Article (CrossRef Link) 
[9] M. Sheng, L. Wang, X. J. Wang, Y. Zhang, C. Xu, and J. D. Li, "Energy efficient beamforming in MISO heterogeneous cellular networks with wireless information and power transfer," IEEE Journal on Selected Areas in Communications, vol. 34, no. 4, pp. 954-968, Apr. 2016. Article (CrossRef Link)

[10] Q. J. Shi, C. Peng, W. Q. Xu, M. Y. Hong, and Y. L. Cai, "Energy efficiency optimization for MISO SWIPT systems with zero-forcing beamforming," IEEE Transactions Signal Processing, vol. 64, no. 4, pp. 842-854, Feb. 2016. Article (CrossRef Link)

[11] F. Yuan, S. Jin, Y. Huang, K. K. Wong, Q. T. Zhang, and H. Zhu, "Joint wireless information and energy transfer in massive distributed antenna systems," IEEE Communications Magazine, vol. 53, no. 6, pp. 109-116, June 2015. Article (CrossRef Link)

[12] D. W. K. Ng and R. Schober, "Secure and green SWIPT in distributed antenna networks with limited backhaul capacity," IEEE Transactions on Wireless Communications, vol. 14, no. 9, pp. 5082-5097, Sep. 2015. Article (CrossRef Link)

[13] F. Yuan, S. Jin, K. K. Wong, J. Zhao, and H. Zhu, "Wireless information and power transfer design for energy cooperation distributed antenna systems," IEEE Access, vol. 5, pp. 8094-8105, 2017. Article (CrossRef Link)

[14] Y. W. Huang, M. Y. Liu, and Y. Liu, "Energy-efficient SWIPT in IoT distributed antenna systems," IEEE Internet of Things Journal, vol. 5, no. 4, pp. 2646-2656, 2018. Article (CrossRef Link)

[15] J. Chu, X. Yu, H. Li, Q. Zhu, and X. Yu, "Energy-efficent optimization scheme for SWIPT-based distributed antenna system," in Proc. of the $10^{\text {th }}$ International Conference on Wireless Communications and Signal Processing (WCSP'2018), pp. 1-5, 2018. Article (CrossRef Link)

[16] H. Kim, S. R. Lee, C. Song, and I. Lee, "Optimal power allocation for energy efficiency maximization in distributed antenna systems," in Proc. of IEEE International Conference on Communications, pp. 5769-5773, 2013. Article (CrossRef Link)

[17] X. Chen, X. Xu, and X. Tao, "Energy efficient power allocation in generalized distributed antenna system," IEEE Communication Letters, vol. 16, no. 7, pp. 1002-1025, July 2012. Article (CrossRef Link)

[18] S. Cui, A. J. Goldsmith, and A. Bahai, "Energy-efficiency of MIMO and Cooperative MIMO techniques in sensor network," IEEE Journal on Selected Areas in Communications, vol. 22, no. 6, pp.1089-1098, 2004. Article (CrossRef Link)

[19] S. Boyd and L. Vandenberghe, Convex Optimization, UK: Cambridge University Press, 2004.

[20] Karush-Kuhn-Tucker Conditions. [Online]. Available: https://encyclopediaofmath.org/wiki/ karush-kuhn-tucker_conditions

[21] W. Yu, L. Musavian, and Q. Ni, "Tradeoff analysis and joint optimization of link-layer energy efficiency and effective capacity toward green communications," IEEE Transactions on Wireless Communications, vol. 15, no. 5, pp. 3339-3353, 2016. Article (CrossRef Link)

[22] L. Musavian and T. Le-Ngoc, "Energy-efficient power allocation over Nakagmi-m fading channels under delay-outage constraints," IEEE Transactions on Wireless Communications, vol. 13, no. 8, pp. 4081-4091, 2014. Article (CrossRef Link)

[23] S. Cui, A. J. Goldsmith, and A. Bahai., "Energy-constrained modulation optimization," IEEE Transactions on Wireless Communications, vol. 4, no. 5, pp. 2349-2360, 2005. Article (CrossRef Link) 

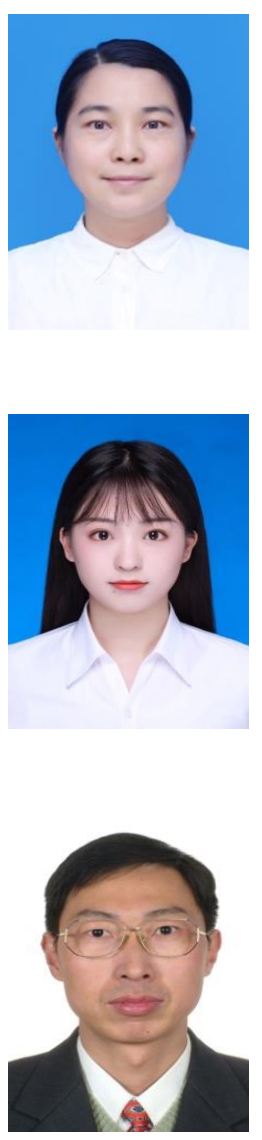

Weiye Xu received the B.S. degree in Communication Engineering and the M.S. degree in Communication and Information Systems from Hohai University, Nanjing, China, in 2001 and 2007, respectively. She received Ph.D. degree in Information and Communication Engineering from Army Engineering University of PLA, Nanjing, China, in 2019. During 2019-2020, she serves as a Visiting Scholar with the School of Informatics, University of Leicester, U.K. She is currently a associate professor with the School of Information and Communication Engineering, Nanjing Institute of Technology, Nanjing, China. Her research interests emphasize on digtial communication, MIMO technique, distributed antenna systems.

Junya Chu received her B.S. degree in information engineering from Nanjing University of Aeronautics and Astronautics. She is currently working towards the M.Sc. degree at Nanjing University of Aeronautics and Astronautics.

Xiangbin Yu received his Ph.D. in Communication and Information Systems in 2004 from National Mobile Communications Research Laboratory at Southeast University, China. He is currently a Full Professor with the Nanjing University of Aeronautics and Astronautics, China. From 2014 to 2015, he worked as a Visiting Scholar in the Electrical and Computer Engineering, University of Delaware, USA. He has been Senior Member of the Chinese Institute of Electronics since 2012. Dr. Yu served as a technical program committee member of the 2006 and 2017 IEEE Global Telecommunications Conference, and the 2015 and 2018 IEEE International Conference on Communications. He is also a Reviewer for several journals. His research interests include distributed MIMO, mmWave communication, precoding, and green communication.

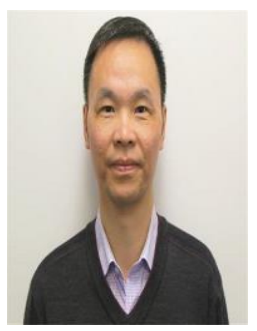

Huiyu Zhou received the B.Eng. degree in radio.technology from Huazhong University of Science and Technology, China, the M.Sc. degree in biomedical engineering from University of Dundee,U.K., and the Ph.D. degree in computer vision from Heriot-Watt University, Edinburgh, U.K. He is currently a Professor with the School of Informatics, University of Leicester, U.K. He has authored or coauthoredover 300 peer-reviewed papers in the field. His research work has been or is being supported byU.K. EPSRC, MRC, EU, Royal Society, Leverhulme Trust, Puffin Trust, Invest NI, and industry. 